\title{
Patterns and Severity of Motorcycle Accidents Injuries at Tanta University Emergency Hospital
}

\author{
Nadia E. Helal ${ }^{1}$, Mohamed A. Shama ${ }^{2}$, and Samah M. Elbastawesy ${ }^{1}$ \\ ${ }^{1}$ Department of Forensic Medicine and Clinical Toxicology, Faculty of Medicine, Tanta University, Tanta, Egypt. \\ ${ }^{2}$ Department of Emergency Medicine and Traumatology, Faculty of Medicine, Tanta University, Tanta, Egypt.
}

\begin{abstract}
Background: In last few years especially during the era of COVID 19 pandemic, motorcycle use has been widely increased either as a mode of transport or for deliveries. However, this increase leads to proportionate increase in number of traffic accidents. Aim of the study: to analyze patterns and severity of injuries in patients of motorcycle accidents and evaluate possible associated factors. Methods: This prospective study was performed at Tanta University Emergency Hospital including all victims of motorcycle accidents during the period from the $15^{\text {th }}$ February 2021 to the $15^{\text {th }}$ of August 2021.Patients were classified according to their position during the accidents into 3 major groups motorcyclist (motorcycle driver), passenger (motorcycle backseat rider) or pedestrian. Data of demographic, injury circumstances, pattern and severity of injuries in addition to outcome in these patients were reported.

Results: The majority of motorcycle accidents occurred in young middle aged males. Frontal collision hitting of a vehicle or pedestrians in agriculture road and over speed were the main injury circumstances. Head injuries were the most commonly reported injuries resulting in higher mortality rate. Severity of injuries was higher in motorcyclists and was strongly associated with over speed together with absence of helmets or personal protective measures. Conclusions: Our study shows that; head injuries were the most commonly reported injuries in motorcycle accidents patients. Over speed and negligence of helmets wear were associated with more severe injuries.
\end{abstract}

Received in original form: 17 August 2021

Accepted in a final form: 9 November 2021

Key words Motorcycle accidents, injuries pattern, severity, Helmet use

\section{Introduction}

$\mathrm{R}$ oad traffic accidents (RTAs) were reported by World Health Organization (WHO) as the 8th cause of death in 2016, causing about 1.35 million deaths every year. Egypt was reported to be one of the highest countries with RTAs rate in Africa and Middle East as stated by statistics of WHO, (2018). This high incidence of RTAs in Egypt may be due to numerous reasons including poor infrastructure and low quality of the majority of the roads. Additionally, the defect in road maintenance regularly and overcrowded traffic with trucks, motor vehicles, motorcycles, buses, and pedestrians may add factors (Fouda et al., 2017). El Bakash et al., (2016) in Egypt, declared that, motorcycle injuries are the second most common road traffic- related injuries. Motorcycles have been extensively used as mode of transport and in commercial delivery work (Aristizábal et al., 2012). Increase usage of motorcycles particularly in large urban areas could be explained by increase in fuel cost, intense traffic and possibly the low price of motorcycles compared to other modes of transportations (Carrasco et al., 2012). Motorcycle delivery work has been increased during last 2 years due to spread of COVID 19 pandemic. The universal very rapid spread of COVID 19 had obligated many countries worldwide to lockdown intervals with increase motorcycle delivery work during these intervals (Zafri et al., 2021). Motorcycles are wellthought-out to be the most unsafe means of transportation because of risk of direct energy transmission to either motorcyclists, riders and/or backseat passengers (CDC, 2012). Severity of motorcycle crash injuries could be dependent on different factors related to either motorcycle riders, environmental conditions, vehicle factors, state of roads and or even multifactorial (Oluwadiya et al., 2009; Sanyang et al., 2017).The motorcyclists who lack proper training, operate without a driving license and safety protective measure as helmet usually are at higher risk of RTAs. Driving under effect of drugs and alcohol may play a role also in such collision. Different studies reported that using helmets has decreased the severity of motorcycle injuries and the incidence of related deaths (Chichom-Mefire et al., 2015). Pedestrian's injuries are also common by motorcycles crash. Less precaution taken by pedestrian while crossing roads or walking along the roadside may be risky. Moreover, low public awareness about road use, less walking on pavement and deficiency of footpaths have increased the incidence of pedestrians injuries in 
RTAs (Jackson and Mello, 2013; Yousaf et al., 2013). The knowledge about different injury patterns in motorcycle accidents (MCA) would be essential for clinical management of the patients and may also help in distinguishing motorcyclist from riders or pedestrians in motorcycle crashes. This research aimed to analyze patterns and severity of injuries in patients of motorcycle accidents and evaluate possible associated factors.

\section{Patients and Methods}

This prospective observational research was performed at Tanta University Emergency Hospital during the period from the $15^{\text {th }}$ February 2021 to the $15^{\text {th }}$ of August 2021. The study was conducted after obtaining approval of the medical research ethical committee of Tanta Faculty of Medicine (approval number, $34455 / 2 / 21$ ). Data were obtained from the patient him/herself or guardians after obtaining a written informed consent from the patient or guardians if not compatible. Some injuries photographs were taken from patients after obtaining his/her consent. The confidentiality of patients' data was preserved by using coding numbers.

\section{Inclusion criteria:}

All victims of motorcycle accidents admitted to Tanta University Emergency Hospital during 6 months' study period were included in the study.

\section{Exclusion criteria:}

Any patient admitted to Tanta University Emergency Hospital due to any cause rather than motorcycle accidents and any missed patients' records data were excluded from the study.

\section{METHODS:}

Patients were classified according to their position during the accidents into 3 major groups: motorcyclist (motorcycle driver), passenger (motorcycle back seat rider) or pedestrian.

The following data have been recorded for every patient:

Demographic data: it includes (age, sex, residence, occupation, special habits as smoking and substance abuse), in addition to delay time till arrival to hospital.

Injury circumstances: including, time of injury (day or nighttime), side of impact: (front, rear or side), accident site (super highway, agricultural road, residential or usual town roads). Use of motor cycle protective gear (helmet, jacket, pants, suits and boots), mechanism of injury (skidding of vehicle, roll over, hit by another vehicle, or tire burst) and accident cause (bad road conditions, over speed, reckless driving, sleepiness and mobile use while driving) also were included.

Vital date/consciousness level: Glasgow Coma Scale (GCS) at admission and clinical stability of the patients were assessed.

Pattern and distribution of injuries were recorded: Craniofacial, neck, thoracic, abdominopelvic, limbs and spine injuries.
Severity of injuries: Assessment of injury severity using injury severity score (ISS). This score depends on system of scoring of anatomical injuries of body regions and finally provides a total score for patients with multiple injuries. The ISS score ranged from 0 to 75 and strongly associated with mortality, morbidity, hospital stay and further measures of severity. Major trauma is well-thought-out when ISS> 15 score (Javali et al., 2019). So, patient's injuries were classified into minor and major injuries according to ISS (ISS $<15$ in minor while ISS $>$ or $=15$ in major injuries).

Outcome of patients: primary outcome measures (Improved, died or complicated). Secondary outcome measures (Admission in intensive care unit (ICU), need for intubation and mechanical ventilation, operative intervention and duration of hospital stay).

STATISTICAL ANALYSIS

The collected data were entered into the Statistical Package for Social Sciences (IBM SPSS Statistics), version 26 (IBM Corp., Armonk, N.Y., USA). The Shapiro-Wilk test for normality showed that the continuous numerical variables did not follow the normal distribution. Therefore, these variables were summarized as the median and interquartile range (IQR), and comparisons were performed using the Kruskal-Wallis test (followed by a post-hoc test if significant). Categorical variables were presented as frequencies. Pearson's Chi-square tests for independence, Fisher's exact test, or Fisher-FreemanHalton exact test were used to inspect the association between two categorical variables as appropriate. A pvalue $<0.05$ was adopted to interpret the significance of the statistical tests (Hinkle et al., 2003).

\section{Results}

Seventy motorcycle accidents patients were included in the current study. They were classified according to their position during motorcycle crash into 3 major groups: motorcyclist group (40 patients, 57.1\%), pedestrians (20 patients, $28.6 \%)$ and backseat passenger group (10 patients, 14.3\%) figure (1).

Table (1): illustrates that median age in motorcycle accidents patients was 22 (about 22.5 in motorcyclist, 36 in backseat passengers and 19 in pedestrians) with no significant difference between the 3 studied groups as regard sociodemographic data as age, residence, special habits, or delayed hospitalization time $(p>0.05)$. However, incidence of motorcycle accidents injuries was higher in males compared to females in the 3 groups. Males accounted for $91.4 \%$ of all patients with marked significant difference $(p<0.05)$. All motorcyclists were only male. Delay time to reach emergency department ranged from half an hour to 72 hours with a median one hour in all groups

Table (2): demonstrates different factors related to motorcycle accidents. Day accidents were significantly higher in motorcyclist and backseat passengers, while night accidents were significantly higher in pedestrians $(\mathrm{p}<0.05)$. Impact site also showed significant difference in the 3 groups $(p<0.05)$ with highest incidence of frontal collision crash being significantly higher in motorcyclists. Concerning 
accident cause, over speed accounted for the majority of MCAs in the current study without statistical difference observed among the studied groups ( $\mathrm{p}>0.05)$. Mechanisms of accidents show high significant difference in the study $(\mathrm{p}<0.05)$. Hitting or being hit by a vehicle represent the major mechanism of the accidents in motorcyclist $(40 \%)$ and pedestrian groups $(100 \%)$, while motorcycle skidding was the only mechanism in backseat passenger group (100\%). Regarding accident site, it was observed that majority of MCAs occurred in the agricultural road (41\%).

Table (3): shows some clinical data in the studied groups. The majority of studied patients were clinically stable with no significant difference between the studied groups as regard their clinical stability and consciousness level assessed by GCS(p>0.05). Range of ISS was from (4-52). Median ISS was significantly differed in the three studied groups and was significantly higher in motorcyclist $(25)(\mathrm{p}<0.05)$.

Table (4): represents distribution and pattern of injuries in the studied groups. Craniofacial injuries were the highest reported injuries in $82.9 \%$ of patients. Different facial injuries (abrasions, contusions, lacerations and even fracture bone) in addition to meningeal hemorrhage were the commonest type of craniofacial injuries (70\% and $40 \%$ respectively). As regard to chest, abdominopelvic and extremities injuries they were reported in $51.4 \%, 38.6 \%$ and $32.9 \%$ of patients, respectively. The most commonly reported chest injuries were lung contusion and pneumothorax (31.4\% and $20 \%$ respectively). Splenic injuries together intraperitoneal free fluids were the mostly reported abdominopelvic injuries $(21.4 \%$ and $25.7 \%$ respectively). Fracture upper limbs were reported more than lower limb fractures $(22.9 \%$ and $18.6 \%$ respectively). In motorcyclist craniofacial injuries were the commonest injuries $(82.5 \%)$ followed by abdominopelvic injuries (50\%). Whereas, in backseat passenger and pedestrians' chest injuries $(50 \%$ and $70 \%$ respectively) were the second most common injuries after craniofacial injuries $(70 \%$ and $90 \%$ respectively). Figure $2,3 \& 4$ show examples of some reported injuries in motorcycle accidents patients in the current study.

Table (5): illustrates primary and secondary outcome (previously explained in methodology) in motorcycle accidents patients. Half number of patients discharged with further follow up. The overall mortality rate in the current study was $21 \%$ and the highest was among motorcyclists (60\% of all deaths). It was noted that about $14.3 \%$ of patients discharged with permanent infirmity in the form of splenectomy, skull bone defect, nerve palsy, amputated limb or finger or even combination of more than one of them in the same patient as in figure (5). About $82.9 \%$ of patients needed ICU admission majority of them were motorcyclists $(60.3 \%)$. Whereas, only $40 \%$ required either intubation and / or mechanical ventilation. The mean duration of hospital stay was 3.7 days and was significantly longer in motorcyclist than other groups (4.5 days). Nearly, $31.4 \%$ of the studied patients needed operative intervention, about $36.4 \%$ of which reported in pedestrian group.

Table (6): demonstrates correlation of different factors to severity of injuries. Major injuries presented in 52 patients $(74.3 \%)$ versus minor injuries in 18 patients $(25.7 \%)$. Frontal impact, over speed, hitting a vehicle and agriculture road accidents were associated with major injuries but with no significant difference $(\mathrm{p}>0.05)$. In addition, higher percentage of patients with major injuries (about 63.5\%) required ICU admission.

Table (1): Demographic characteristics of motorcycle accidents patients $(n=70)$

\begin{tabular}{|c|c|c|c|c|c|c|c|c|c|c|c|}
\hline & & \multicolumn{8}{|c|}{ Patient type } & \multicolumn{2}{|c|}{ Tests of significance } \\
\hline & & \multirow{2}{*}{\multicolumn{2}{|c|}{$\begin{array}{c}\begin{array}{c}\text { Total } \\
(\mathbf{n}=\mathbf{7 0})\end{array} \\
22.0 \\
{[18.0-} \\
40.0] \\
(2.0-70.0) \\
\end{array}$}} & \multirow{2}{*}{\multicolumn{2}{|c|}{$\begin{array}{c}\begin{array}{c}\text { back seat } \\
\text { passenger } \\
(\mathbf{n}=\mathbf{1 0})\end{array} \\
36.0 \\
{[2.0-55.0]} \\
(2.0-55.0)\end{array}$}} & \multirow{2}{*}{\multicolumn{2}{|c|}{$\begin{array}{c}\begin{array}{c}\text { Motorcyclist } \\
(\mathbf{n}=\mathbf{4 0})\end{array} \\
22.5 \\
{\left[\begin{array}{c}18.0-35.0] \\
(7.0-67.0)\end{array}\right.}\end{array}$}} & \multirow{2}{*}{\multicolumn{2}{|c|}{$\begin{array}{c}\begin{array}{c}\text { Pedestrian } \\
(\mathbf{n}=\mathbf{2 0})\end{array} \\
19.0 \\
{[15.0-51.0]} \\
(2.5-70.0)\end{array}$}} & Test statistic & $\mathbf{p}$ \\
\hline $\begin{array}{c}\text { Age } \\
\text { (years) }\end{array}$ & $\begin{array}{l}\text { Median } \\
\text { [IQR] } \\
\text { (Range) }\end{array}$ & & & & & & & & & $0.423^{\mathrm{a}}$ & 0.810 \\
\hline \multirow{2}{*}{ Gender } & Females & 6 & $8.6 \%$ & 2 & $20.0 \%$ & 0 & $0.0 \%$ & 4 & $20.0 \%$ & \multirow{2}{*}{$9.477^{\mathrm{b}}$} & \multirow{2}{*}{$0.007 *$} \\
\hline & Males & 64 & $91.4 \%$ & 8 & $80.0 \%$ & 40 & $100.0 \%$ & 16 & $80.0 \%$ & & \\
\hline \multirow{2}{*}{ Residence } & Rural & 34 & $48.6 \%$ & 5 & $50.0 \%$ & 22 & $55.0 \%$ & 7 & $35.0 \%$ & \multirow{2}{*}{$2.153^{\mathrm{b}}$} & \multirow{2}{*}{0.355} \\
\hline & Urban & 36 & $51.4 \%$ & 5 & $50.0 \%$ & 18 & $45.0 \%$ & 13 & $65.0 \%$ & & \\
\hline \multirow{4}{*}{$\begin{array}{c}\text { Special } \\
\text { habits }\end{array}$} & None & 31 & $44.3 \%$ & 5 & $50.0 \%$ & 16 & $40.0 \%$ & 10 & $50.0 \%$ & \multirow{4}{*}{$3.529^{b}$} & \multirow{4}{*}{0.817} \\
\hline & Addict & 7 & $10.0 \%$ & 0 & $0.0 \%$ & 6 & $15.0 \%$ & 1 & $5.0 \%$ & & \\
\hline & Smoker & 31 & $44.3 \%$ & 5 & $50.0 \%$ & 17 & $42.5 \%$ & 9 & $45.0 \%$ & & \\
\hline & $\begin{array}{l}\text { Smoker } \\
\& \text { addict }\end{array}$ & 1 & $1.4 \%$ & 0 & $0.0 \%$ & 1 & $2.5 \%$ & 0 & $0.0 \%$ & & \\
\hline $\begin{array}{c}\text { Delay time } \\
\text { (hours) }\end{array}$ & $\begin{array}{c}\text { Median } \\
\text { [IQR] } \\
\text { (Range) }\end{array}$ & \multicolumn{2}{|c|}{$\begin{array}{c}1.0 \\
{[0.5-1.3]} \\
{[0.5-72.0]}\end{array}$} & \multicolumn{2}{|c|}{$\begin{array}{c}1.0 \\
{[1.0-1.0]} \\
(0.5-1.0)\end{array}$} & \multicolumn{2}{|c|}{$\begin{array}{c}1.0 \\
{[0.5-1.5]} \\
(0.5-72.0)\end{array}$} & \multicolumn{2}{|c|}{$\begin{array}{c}1.0 \\
{[0.5-2.0]} \\
(0.5-8.0)\end{array}$} & $0.170^{\mathrm{a}}$ & 0.919 \\
\hline
\end{tabular}

a: Kruskal-Wallis test; b: Fisher-Freeman-Halton exact test; IQR: interquartile range; $n$ : number; * significant at $p<0.05 ; \$$ significant difference from other groups. 
Table (2): Factors related to the circumstances of motorcycle accidents $(n=70)$

\begin{tabular}{|c|c|c|c|c|c|c|c|c|c|c|c|}
\hline \multirow{4}{*}{ Time } & \multirow{4}{*}{$\begin{array}{l}\text { Day } \\
\text { Night }\end{array}$} & \multicolumn{8}{|c|}{ Patient type } & \multicolumn{2}{|c|}{$\begin{array}{c}\text { Tests of } \\
\text { significance }\end{array}$} \\
\hline & & \multicolumn{2}{|c|}{$\begin{array}{c}\text { Total } \\
(n=70)\end{array}$} & \multicolumn{2}{|c|}{$\begin{array}{c}\text { Back seat } \\
\text { passenger } \\
(\mathbf{n}=10)\end{array}$} & \multicolumn{2}{|c|}{$\begin{array}{l}\text { Motorcyclist } \\
\quad(n=40)\end{array}$} & \multicolumn{2}{|c|}{$\begin{array}{l}\text { Pedestrian } \\
(n=20)\end{array}$} & $\begin{array}{c}\text { Test } \\
\text { statistic }\end{array}$ & $\mathbf{p}$ \\
\hline & & 41 & $58.6 \%$ & 10 & $100.0 \% \$$ & 22 & $55.0 \%$ & 9 & $45.0 \%$ & & \\
\hline & & 29 & $41.4 \%$ & 0 & $0.0 \%$ & 18 & $45.0 \%$ & 11 & $55.0 \%$ & $9.742^{\mathrm{a}}$ & $0.007^{*}$ \\
\hline \multirow{4}{*}{$\begin{array}{c}\text { Impact side } \\
\text { (front/rear/side) }\end{array}$} & Frontal & 40 & $57.1 \%$ & 5 & $50.0 \%$ & 26 & $65.0 \%$ & 9 & $45.0 \%$ & \multirow{4}{*}{$16.580^{\mathrm{a}}$} & \multirow{4}{*}{$0.004 *$} \\
\hline & Lateral & 15 & $21.4 \%$ & 5 & $50.0 \%$ & 5 & $12.5 \%$ & 5 & $25.0 \%$ & & \\
\hline & Rear & 8 & $11.4 \%$ & 0 & $0.0 \%$ & 2 & $5.0 \%$ & 6 & $30.0 \%$ & & \\
\hline & Roll over & 7 & $10.0 \%$ & 0 & $0.0 \%$ & 7 & $17.5 \%$ & 0 & $0.0 \%$ & & \\
\hline \multirow{4}{*}{ Accident cause } & $\begin{array}{l}\text { Bad road } \\
\text { conditions }\end{array}$ & 23 & $32.9 \%$ & 5 & $50.0 \%$ & 12 & $30.0 \%$ & 6 & $30.0 \%$ & \multirow{4}{*}{$5.733^{\mathrm{a}}$} & \multirow{4}{*}{0.423} \\
\hline & Over speed & 31 & $44.3 \%$ & 5 & $50.0 \%$ & 19 & $47.5 \%$ & 7 & $35.0 \%$ & & \\
\hline & $\begin{array}{l}\text { Reckless } \\
\text { driving }\end{array}$ & 13 & $18.6 \%$ & 0 & $0.0 \%$ & 8 & $20.0 \%$ & 5 & $25.0 \%$ & & \\
\hline & Sleepiness & 3 & $4.3 \%$ & 0 & $0.0 \%$ & 1 & $2.5 \%$ & 2 & $10.0 \%$ & & \\
\hline \multirow{4}{*}{$\begin{array}{l}\text { Mechanism of } \\
\text { accident }\end{array}$} & $\begin{array}{c}\text { Hit with/by } \\
\text { vehicle }\end{array}$ & 36 & $51.4 \%$ & 0 & $0.0 \% \$$ & 16 & $40.0 \%$ & 20 & $100.0 \%$ & \multirow{4}{*}{$52.337^{\mathrm{a}}$} & \multirow{4}{*}{$<0.001 *$} \\
\hline & $\begin{array}{l}\text { Hit an electric } \\
\text { pole }\end{array}$ & 3 & $4.3 \%$ & 0 & $0.0 \%$ & 3 & $7.5 \%$ & 0 & $0.0 \%$ & & \\
\hline & $\begin{array}{l}\text { Rollover } \\
\text { accident }\end{array}$ & 16 & $22.9 \%$ & 0 & $0.0 \%$ & 16 & $40.0 \% \$$ & 0 & $0.0 \%$ & & \\
\hline & Skidding & 15 & $21.4 \%$ & 10 & $100.0 \% \$$ & 5 & $12.5 \%$ & 0 & $0.0 \%$ & & \\
\hline \multirow{5}{*}{ Accident site } & $\begin{array}{l}\text { Agricultural } \\
\text { road }\end{array}$ & 29 & $41.4 \%$ & 2 & $20.0 \%$ & 15 & $37.5 \%$ & 12 & $60.0 \%$ & \multirow{5}{*}{$16.617^{\mathrm{a}}$} & \multirow{5}{*}{$0.016^{*}$} \\
\hline & Highway road & 12 & $17.1 \%$ & 5 & $50.0 \%$ & 7 & $17.5 \%$ & 0 & $0.0 \%$ & & \\
\hline & $\begin{array}{l}\text { Residential } \\
\text { area }\end{array}$ & 6 & $8.6 \%$ & 0 & $0.0 \%$ & 5 & $12.5 \%$ & 1 & $5.0 \%$ & & \\
\hline & Super highway & 12 & $17.1 \%$ & 3 & $30.0 \%$ & 7 & $17.5 \%$ & 2 & $10.0 \%$ & & \\
\hline & $\begin{array}{l}\text { Usual town } \\
\text { roads }\end{array}$ & 11 & $15.7 \%$ & 0 & $0.0 \%$ & 6 & $15.0 \%$ & 5 & $25.0 \%$ & & \\
\hline
\end{tabular}

a: Fisher-Freeman-Halton exact test; $n:$ number; * significant at $p<0.05 ; \$$ significant difference from other groups.

Table (3): Clinical data of motorcycle accidents patients $(\mathbf{n}=70)$

\begin{tabular}{|c|c|c|c|c|c|c|c|c|c|c|c|}
\hline \multirow{5}{*}{$\begin{array}{l}\text { Clinical } \\
\text { stability }\end{array}$} & \multirow[b]{3}{*}{ Shocked } & \multicolumn{8}{|c|}{ Patient type } & \multicolumn{2}{|c|}{$\begin{array}{c}\text { Tests of } \\
\text { significance }\end{array}$} \\
\hline & & \multicolumn{2}{|c|}{$\begin{array}{c}\text { Total } \\
(n=70)\end{array}$} & \multicolumn{2}{|c|}{$\begin{array}{c}\text { Back seat } \\
\text { passenger } \\
(\mathbf{n}=10)\end{array}$} & \multicolumn{2}{|c|}{$\begin{array}{l}\text { Motorcyclist } \\
\quad(\mathrm{n}=\mathbf{4 0})\end{array}$} & \multicolumn{2}{|c|}{$\begin{array}{l}\text { Pedestrian } \\
(\mathbf{n}=\mathbf{2 0})\end{array}$} & $\begin{array}{c}\text { Test } \\
\text { statistic }\end{array}$ & $\mathbf{p}$ \\
\hline & & 1 & $1.4 \%$ & 0 & $0.0 \%$ & 1 & $2.5 \%$ & 0 & $0.0 \%$ & \multirow{3}{*}{$3.793^{\mathrm{a}}$} & \multirow{3}{*}{0.471} \\
\hline & Stable & 43 & $61.4 \%$ & 8 & $80.0 \%$ & 25 & $62.5 \%$ & 10 & $50.0 \%$ & & \\
\hline & Unstable & 26 & $37.1 \%$ & 2 & $20.0 \%$ & 14 & $35.0 \%$ & 10 & $50.0 \%$ & & \\
\hline GCS & $\begin{array}{c}\text { Median } \\
\text { [IQR] } \\
\text { (Range) }\end{array}$ & \multicolumn{2}{|c|}{$\begin{array}{c}11 \\
{[5-14]} \\
(3-15)\end{array}$} & \multicolumn{2}{|c|}{$\begin{array}{c}13 \\
{[11-15]} \\
(11-15)\end{array}$} & \multicolumn{2}{|c|}{$\begin{array}{c}11 \\
{[6-14]} \\
(3-15)\end{array}$} & \multicolumn{2}{|c|}{$\begin{array}{c}8 \\
{[3-13]} \\
(3-15)\end{array}$} & $5.776^{\mathrm{b}}$ & 0.056 \\
\hline ISS & $\begin{array}{c}\text { Median } \\
\text { [IQR] } \\
\text { (Range) }\end{array}$ & \multicolumn{2}{|c|}{$\begin{array}{c}24 \\
{[14-29]} \\
(4-52)\end{array}$} & \multicolumn{2}{|c|}{$\begin{array}{c}19 c \\
{[4-20]} \\
(4-29)\end{array}$} & \multicolumn{2}{|c|}{$\begin{array}{c}25 \mathrm{~d}, \mathrm{e} \\
{[19-33]} \\
(9-52)\end{array}$} & \multicolumn{2}{|c|}{$\begin{array}{c}19 c \\
{[9-27]} \\
(8-49)\end{array}$} & $6.183^{\mathrm{b}}$ & $0.045^{*}$ \\
\hline
\end{tabular}

a: Fisher-Freeman-Halton exact test; $b$ : Kruskal-Wallis test; $c$ : significant difference from motorcyclists, d: significant difference from passengers; : significant difference from pedestrians; IQR: interquartile range; n: number, ISS: Injury Severity Score, GCS: Glasgow Coma Scale. 
Table (4): Pattern and distribution of injuries in motorcycle accidents patients $(n=70)$

\begin{tabular}{|c|c|c|c|c|c|c|c|c|c|c|}
\hline \multirow[b]{3}{*}{ Craniofacial } & \multicolumn{8}{|c|}{ Patient type } & \multicolumn{2}{|c|}{ Tests of significance } \\
\hline & \multicolumn{2}{|c|}{$\begin{array}{c}\text { Total } \\
(n=70)\end{array}$} & \multicolumn{2}{|c|}{$\begin{array}{c}\text { back seat } \\
\text { passenger } \\
(\mathbf{n}=\mathbf{1 0})\end{array}$} & \multicolumn{2}{|c|}{$\begin{array}{l}\text { Motorcyclist } \\
\quad(n=40)\end{array}$} & \multicolumn{2}{|c|}{$\begin{array}{c}\text { Pedestrian } \\
(\mathbf{n}=\mathbf{2 0})\end{array}$} & \multirow{2}{*}{$\begin{array}{c}\begin{array}{c}\text { Test } \\
\text { statistic }\end{array} \\
1.929^{\mathrm{a}}\end{array}$} & \multirow{2}{*}{$\begin{array}{c}\mathbf{p} \\
0.405\end{array}$} \\
\hline & 58 & $82.9 \%$ & 7 & $70.0 \%$ & 33 & $82.5 \%$ & 18 & $90.0 \%$ & & \\
\hline Scalp lesions & 9 & $12.9 \%$ & 2 & $20.0 \%$ & 4 & $10.0 \%$ & 3 & $15.0 \%$ & $1.223^{\mathrm{a}}$ & 0.686 \\
\hline Fracture skull & 25 & $35.7 \%$ & 5 & $50.0 \%$ & 12 & $30.0 \%$ & 8 & $40.0 \%$ & $1.718^{\mathrm{a}}$ & 0.443 \\
\hline Meningeal haemorrhage & 28 & $40.0 \%$ & 5 & $50.0 \%$ & 16 & $40.0 \%$ & 7 & $35.0 \%$ & $0.691^{\mathrm{a}}$ & 0.699 \\
\hline Brain edema & 11 & $15.7 \%$ & 0 & $0.0 \%$ & 5 & $12.5 \%$ & 6 & $30.0 \%$ & $4.510^{\mathrm{a}}$ & 0.094 \\
\hline Intracerebral hematoma & 6 & $8.6 \%$ & 0 & $0.0 \%$ & 5 & $12.5 \%$ & 1 & $5.0 \%$ & $1.276^{\mathrm{a}}$ & 0.585 \\
\hline Brain contusion & 26 & $37.1 \%$ & 5 & $50.0 \%$ & 12 & $30.0 \%$ & 9 & $45.0 \%$ & $2.206^{\mathrm{a}}$ & 0.349 \\
\hline Pneumoencephalus & 2 & $2.9 \%$ & 0 & $0.0 \%$ & 2 & $5.0 \%$ & 0 & $0.0 \%$ & $1.015^{\mathrm{a}}$ & 0.669 \\
\hline Facial injuries & 49 & $70.0 \%$ & 7 & $70.0 \%$ & 29 & $72.5 \%$ & 13 & $65.0 \%$ & $0.479^{\mathrm{a}}$ & 0.874 \\
\hline Chest & 36 & $51.4 \%$ & 5 & $50.0 \%$ & 17 & $42.5 \%$ & 14 & $70.0 \%$ & $4.030^{\mathrm{a}}$ & 0.136 \\
\hline$\overline{\text { Surgical emphysema }}$ & 1 & $1.4 \%$ & 0 & $0.0 \%$ & 0 & $0.0 \%$ & 1 & $5.0 \%$ & $2.617^{\mathrm{a}}$ & 0.429 \\
\hline Fracture chest bone & 9 & $12.9 \%$ & 3 & $30.0 \%$ & 2 & $5.0 \% \$$ & 4 & $20.0 \%$ & $5.895^{\mathrm{a}}$ & $0.045 *$ \\
\hline Lung contusion & 22 & $31.4 \%$ & 3 & $30.0 \%$ & 11 & $27.5 \%$ & 8 & $40.0 \%$ & $1.058^{\mathrm{a}}$ & 0.585 \\
\hline Pneumothorax & 14 & $20.0 \%$ & 5 & $50.0 \%$ & 8 & $20.0 \%$ & 1 & $5.0 \%$ & $7.674^{\mathrm{a}}$ & $0.017 *$ \\
\hline Hemothorax & 11 & $15.7 \%$ & 3 & $30.0 \%$ & 5 & $12.5 \%$ & 3 & $15.0 \%$ & $1.988^{\mathrm{a}}$ & 0.428 \\
\hline Pulmonary hemorrhage & 2 & $2.9 \%$ & 0 & $0.0 \%$ & 2 & $5.0 \%$ & 0 & $0.0 \%$ & $1.015^{\mathrm{a}}$ & 0.669 \\
\hline Abdominopelvic & 27 & $38.6 \%$ & 2 & $20.0 \%$ & 20 & $50.0 \%$ & 5 & $25.0 \%$ & $4.952^{\mathrm{a}}$ & 0.085 \\
\hline IPFF & 18 & $25.7 \%$ & 0 & $0.0 \%$ & 13 & $32.5 \%$ & 5 & $25.0 \%$ & $4.542^{\mathrm{a}}$ & 0.099 \\
\hline Splenic injuries & 15 & $21.4 \%$ & 2 & $20.0 \%$ & 11 & $27.5 \%$ & 2 & $10.0 \%$ & $2.343^{\mathrm{a}}$ & 0.382 \\
\hline Hepatic injuries & 1 & $1.4 \%$ & 0 & $0.0 \%$ & 1 & $2.5 \%$ & 0 & $0.0 \%$ & $1.231^{\mathrm{a}}$ & 1.000 \\
\hline Pelvic injuries & 7 & $10.0 \%$ & 0 & $0.0 \%$ & 6 & $15.0 \%$ & 1 & $5.0 \%$ & $1.898^{\mathrm{a}}$ & 0.321 \\
\hline Extremities & 23 & $32.9 \%$ & 0 & $0.0 \% \$$ & 15 & $37.5 \%$ & 8 & $40.0 \%$ & $6.344^{\mathrm{a}}$ & $0.044 *$ \\
\hline$\overline{\text { Fracture upper limb }}$ & 16 & $22.9 \%$ & 0 & $0.0 \%$ & 9 & $22.5 \%$ & 7 & $35.0 \%$ & $4.581^{\mathrm{a}}$ & 0.079 \\
\hline Fracture lower limb & 13 & $18.6 \%$ & 0 & $0.0 \%$ & 7 & $17.5 \%$ & 6 & $30.0 \%$ & $3.733^{\mathrm{a}}$ & 0.143 \\
\hline
\end{tabular}

a: Fisher-Freeman-Halton exact test; $n:$ number; * significant at $p<0.05 ; \$$ significant difference from other groups, IPFF: Intra Peritoneal Free Fluid.

Table (5): Outcome in motorcycle accidents patients $(n=70)$

\begin{tabular}{|c|c|c|c|c|c|c|}
\hline & $\begin{array}{c}\text { Total } \\
(\mathbf{n}=\mathbf{7 0}) \\
\end{array}$ & $\begin{array}{l}\text { Motorcyclist } \\
\quad(n=40)\end{array}$ & $\begin{array}{c}\text { passenger } \\
(\mathrm{n}=10)\end{array}$ & $\begin{array}{c}\text { Pedestrian } \\
(n=20)\end{array}$ & $\begin{array}{c}\text { Test of } \\
\text { significance }\end{array}$ & $\mathbf{P}$ \\
\hline Discharged free & $10(14.3 \%)$ & $3(7.5 \%)$ & $3(30.0 \%)$ & $4(20.0 \%)$ & $4.256^{\mathrm{a}}$ & 0.112 \\
\hline $\begin{array}{l}\text { Discharged with } \\
\text { further follow up }\end{array}$ & $35(50.0 \%)$ & $22(55.0 \%)$ & $6(60.0 \%)$ & $7(35.0 \%)$ & $2.600^{b}$ & 0.273 \\
\hline $\begin{array}{l}\text { Discharged with } \\
\text { complication and or } \\
\text { permanent infirmity }\end{array}$ & $10(14.3 \%)$ & $6(15.0 \%)$ & $1(10.0 \%)$ & $3(15.0 \%)$ & $0.195^{\mathrm{a}}$ & 1.000 \\
\hline Died & $15(21.4 \%)$ & $9(22.5 \%)$ & $0(0.0 \%)$ & $6(30.0 \%)$ & $3.590^{\mathrm{a}}$ & 0.132 \\
\hline ICU admission & $58(82.9 \%)$ & $35(87.5 \%)$ & $7(70.0 \%)$ & $16(80.0 \%)$ & $2.140^{\mathrm{a}}$ & 0.365 \\
\hline $\begin{array}{c}\text { Intubation and } \\
\text { mechanical } \\
\text { ventilation } \\
\end{array}$ & $28(40.0 \%)$ & $16(40.0 \%)$ & $5(50.0 \%)$ & $7(35.0 \%)$ & $0.691^{\mathrm{a}}$ & 0.699 \\
\hline $\begin{array}{c}\text { Duration of hospital } \\
\text { stay (days) }\end{array}$ & $\begin{array}{c}3.7 \\
{[2.0-6.9]} \\
(0.1-22.0) \\
\end{array}$ & $\begin{array}{c}4.5^{\mathrm{e}} \\
{[2.2-8.4]} \\
(0.1-22.0) \\
\end{array}$ & $\begin{array}{c}4.4 \\
{[3.5-6.9]} \\
(1.6-15.0) \\
\end{array}$ & $\begin{array}{c}2.8^{\mathrm{d}} \\
{[0.7-4.0]} \\
(0.1-7.0) \\
\end{array}$ & $7.827^{\mathrm{c}}$ & $0.020 *$ \\
\hline $\begin{array}{c}\text { Operative } \\
\text { intervention }\end{array}$ & $22(31.4 \%)$ & $12(30.0 \%)$ & $2(20.0 \%)$ & $8(40.0 \%)$ & $1.255^{\mathrm{a}}$ & 0.545 \\
\hline
\end{tabular}

a: Fisher-Freeman-Halton exact test; b: Pearson's Chi-square test; $n$ : number; * significant at p<0.05; c: KruskalWallis test; $d$ : significant difference from motorcyclists; $e$ : significant difference from pedestrians, ICU: Intensive Care Unit. 
Table (6) Correlation of different factors to severity of injuries $(n=70)$

\begin{tabular}{|c|c|c|c|c|c|c|c|}
\hline & & \multicolumn{4}{|c|}{ Severity } & \multicolumn{2}{|c|}{ Tests of significance } \\
\hline & & \multicolumn{2}{|c|}{$\begin{array}{c}\text { Major } \\
(n=52)\end{array}$} & \multicolumn{2}{|c|}{$\begin{array}{l}\text { Minor } \\
(\mathbf{n}=\mathbf{1 8})\end{array}$} & $\begin{array}{c}\text { Test } \\
\text { statistic }\end{array}$ & $\mathbf{p}$ \\
\hline \multirow{4}{*}{ Impact side } & Frontal & 30 & $57.7 \%$ & 10 & $55.6 \%$ & \multirow{4}{*}{$5.830^{\mathrm{a}}$} & \multirow{4}{*}{0.100} \\
\hline & Lateral & 8 & $15.4 \%$ & 7 & $38.9 \%$ & & \\
\hline & Rear & 7 & $13.5 \%$ & 1 & $5.6 \%$ & & \\
\hline & Roll over & 7 & $13.5 \%$ & 0 & $0.0 \%$ & & \\
\hline \multirow{4}{*}{ Accident cause } & Bad road conditions & 15 & $28.8 \%$ & 8 & $44.4 \%$ & \multirow{4}{*}{$7.019^{\mathrm{a}}$} & \multirow{4}{*}{0.061} \\
\hline & Over speed & 27 & $51.9 \%$ & 4 & $22.2 \%$ & & \\
\hline & Reckless driving & 7 & $13.5 \%$ & 6 & $33.3 \%$ & & \\
\hline & Sleepiness & 3 & $5.8 \%$ & 0 & $0.0 \%$ & & \\
\hline \multirow{4}{*}{$\begin{array}{l}\text { Mechanism of } \\
\text { accident }\end{array}$} & Hit a vehicle & 24 & $46.2 \%$ & 12 & $66.7 \%$ & \multirow{4}{*}{$2.112^{\mathrm{a}}$} & \multirow{4}{*}{0.549} \\
\hline & Hit an electric pole & 3 & $5.8 \%$ & 0 & $0.0 \%$ & & \\
\hline & Rollover accident & 13 & $25.0 \%$ & 3 & $16.7 \%$ & & \\
\hline & Skidding & 12 & $23.1 \%$ & 3 & $16.7 \%$ & & \\
\hline \multirow{5}{*}{ Accident site } & Agricultural road & 21 & $40.4 \%$ & 8 & $44.4 \%$ & \multirow{5}{*}{$6.836^{\mathrm{a}}$} & \multirow{5}{*}{0.128} \\
\hline & High way road & 12 & $23.1 \%$ & 0 & $0.0 \%$ & & \\
\hline & Residential area & 4 & $7.7 \%$ & 2 & $11.1 \%$ & & \\
\hline & Super high way & 7 & $13.5 \%$ & 5 & $27.8 \%$ & & \\
\hline & Usual Town roads & 8 & $15.4 \%$ & 3 & $16.7 \%$ & & \\
\hline \multirow{2}{*}{ ICU } & No & 19 & $36.5 \%$ & 11 & $61.1 \%$ & \multirow{2}{*}{$3.297^{b}$} & \multirow{2}{*}{0.069} \\
\hline & Yes & 33 & $63.5 \%$ & 7 & $38.9 \%$ & & \\
\hline
\end{tabular}

a: Fisher-Freeman-Halton exact test; b: Pearson's Chi-square test; n: number, ICU: Intensive Care Unit.

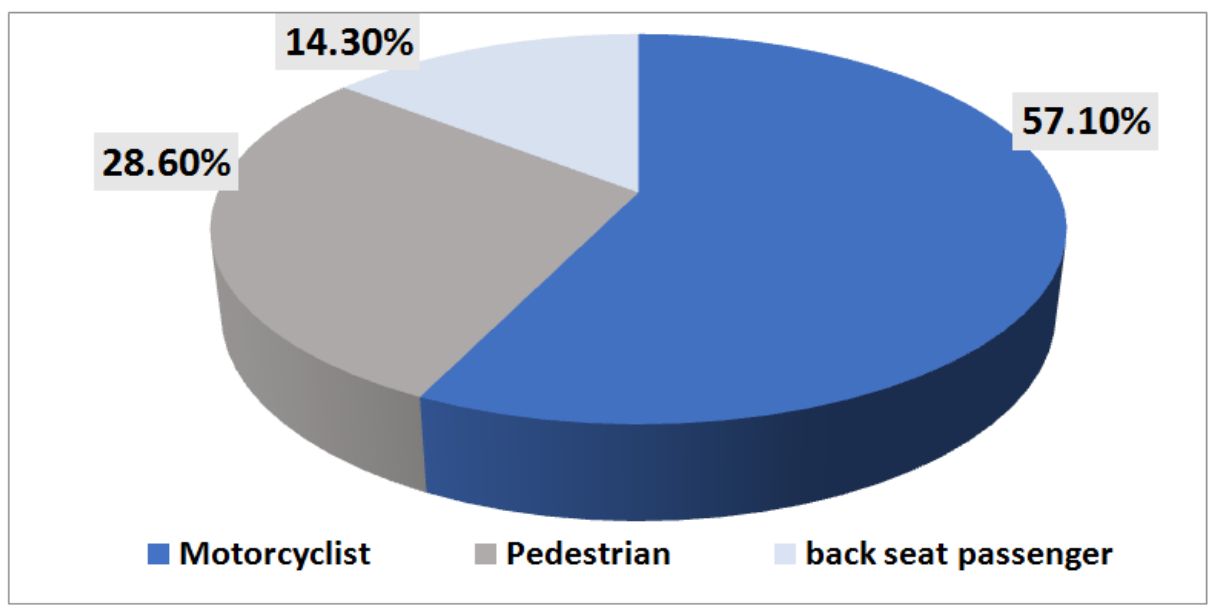

Figure (1): Distribution of the studied motorcycle accidents patients

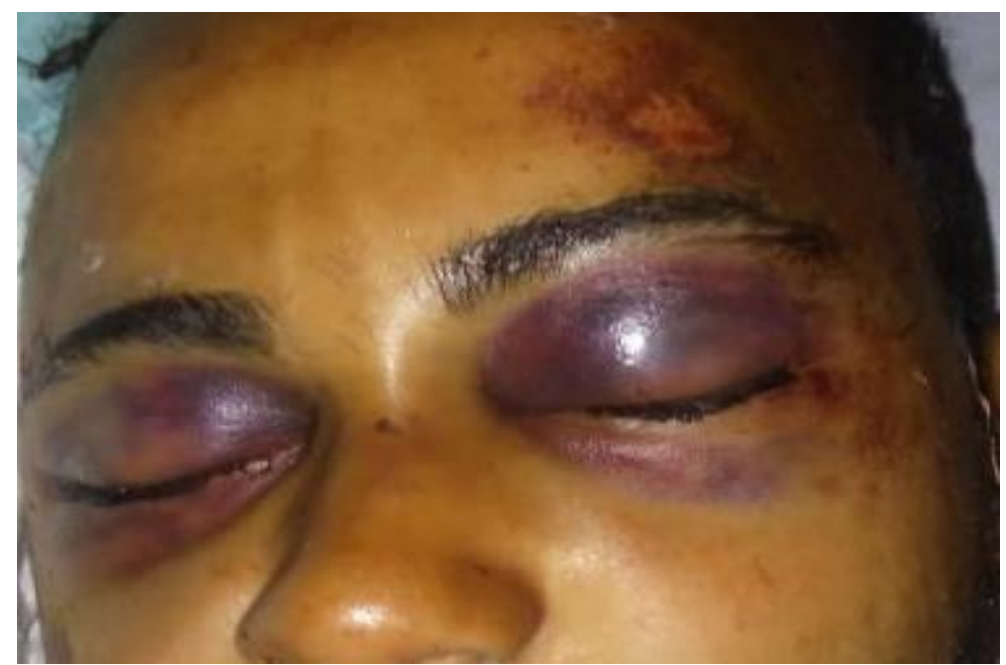

Figure (2): Bilateral black eye (raccoon eyes), facial abrasions, and fractured dislocated nasal septum in motorcycle accident patient (motorcyclist group). 


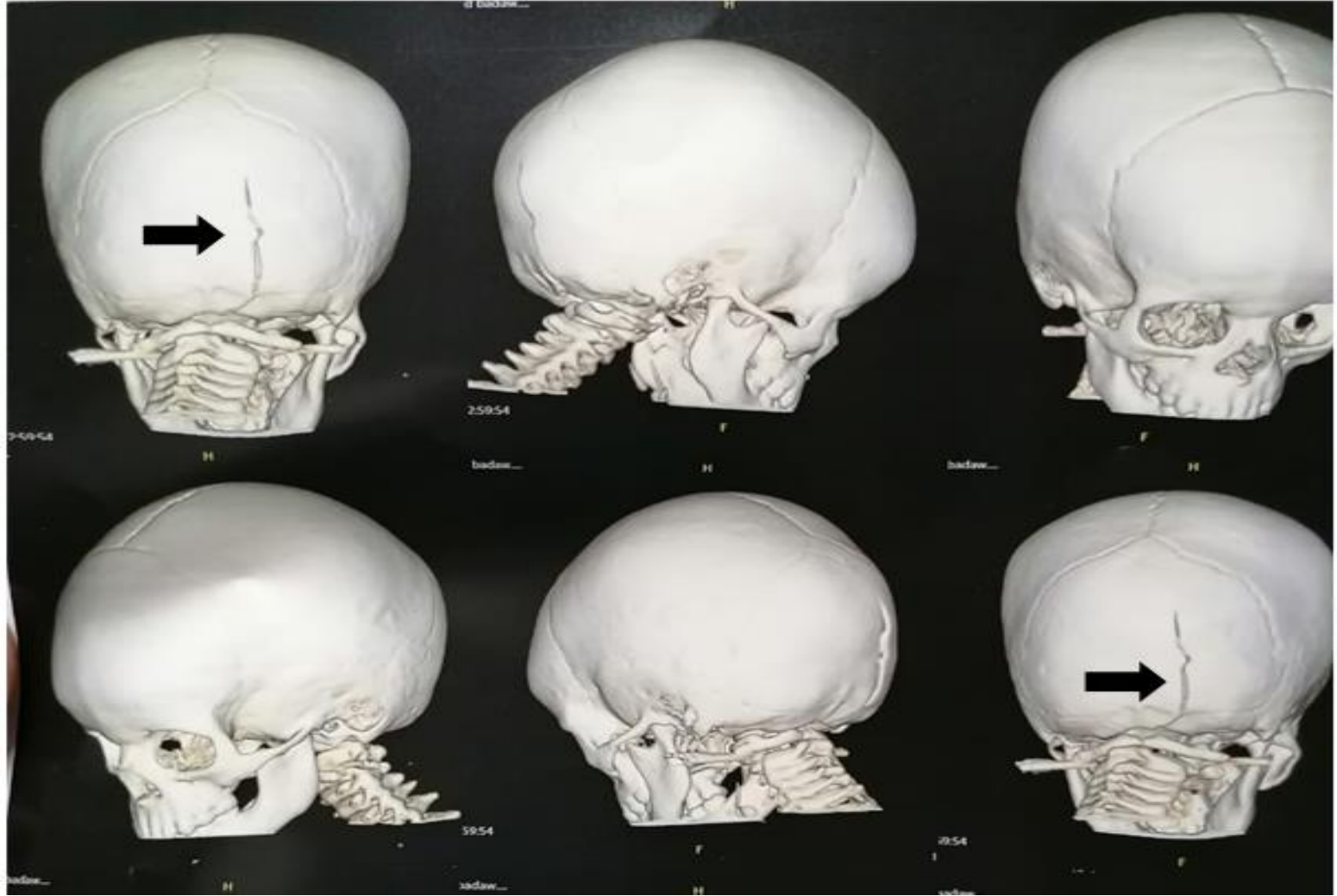

Figure (3): Computerized Tomography (CT) scan of motorcycle accident patient head (Pedestrain group), showing occipital fissure fracture (black arrow).

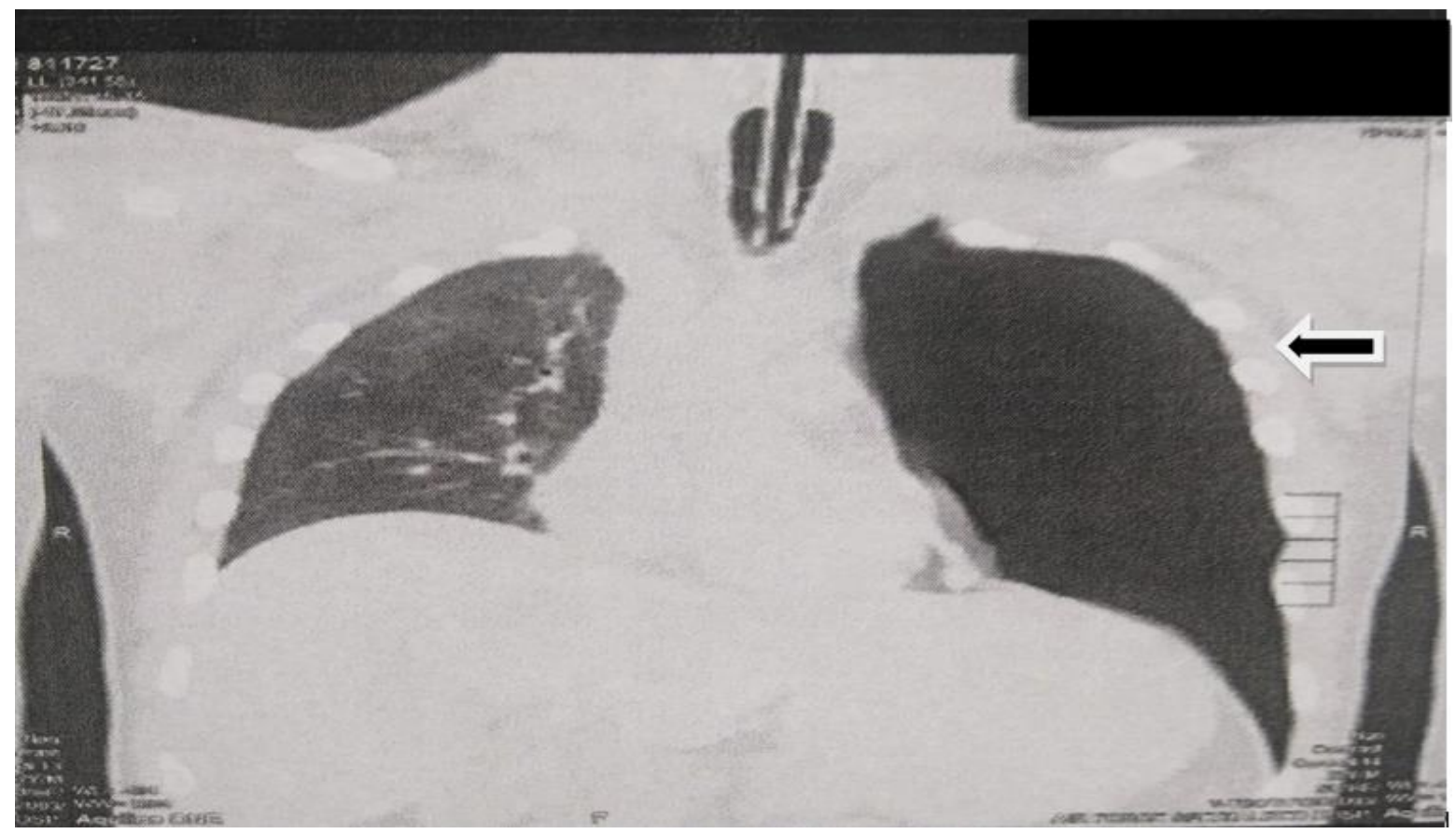

Figure (4): Computerized Tomography (CT) scan of motorcycle accident patient chest (motorcyclists group), showing left lung side pneumothorax (black arrow). 


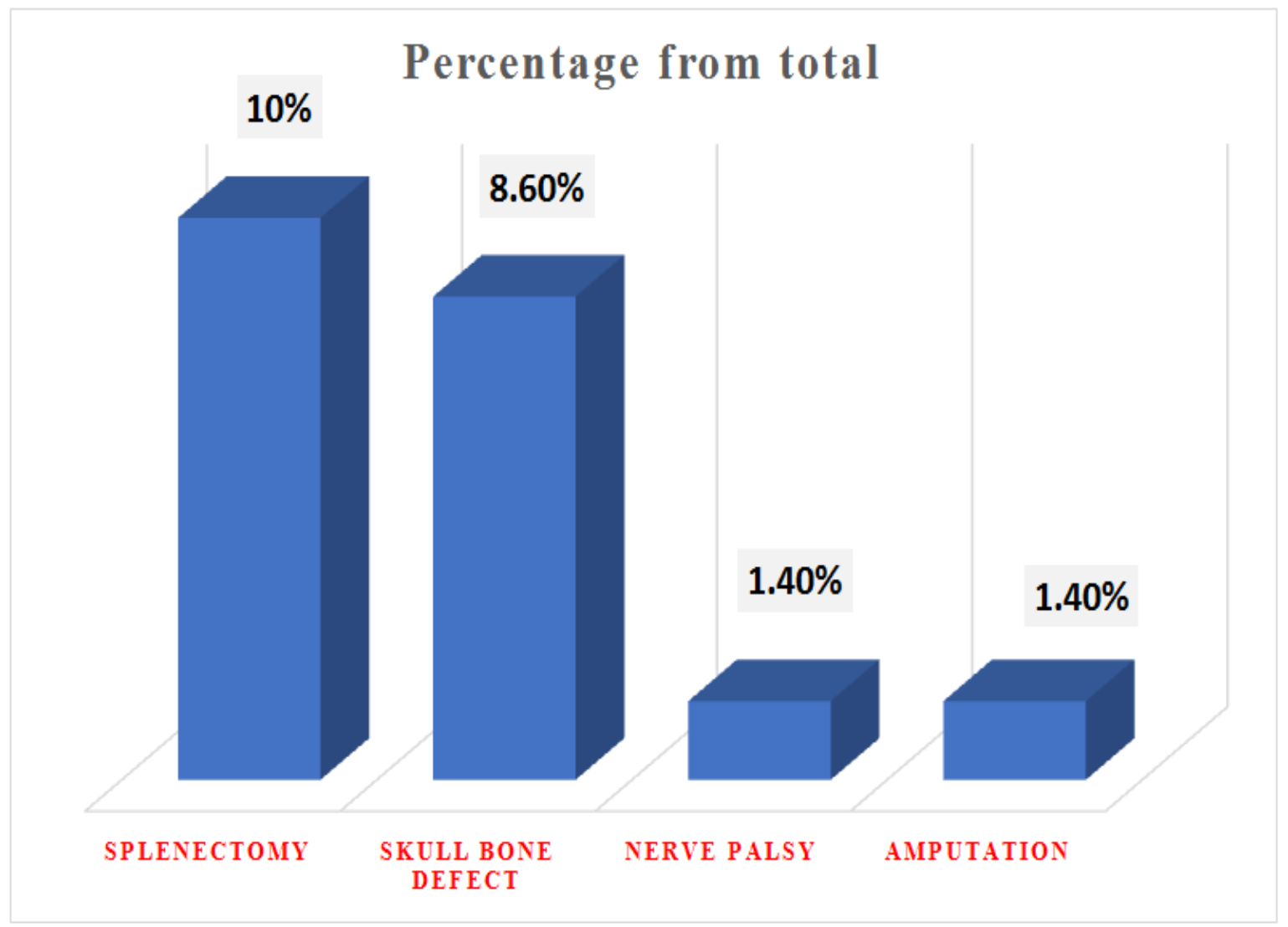

Figure (5): Percentage of different types of permanent infirmity among the studied patients.

\section{Discussion}

According to WHO about one million people die every year due to traffic accidents, and $90 \%$ are concentrated in low developed countries (WHO, 2018). In last few years, especially during the era of COVID 19 pandemic motorcycle usage has been widely increased either as a mode of transport or for deliveries. However, this increase lead to proportionate increase in number of traffic accidents (Bittar et al., 2020). This study aimed to analyze patterns and severity of injuries in patients of motorcycle accidents and evaluate possible associated factors.

The majority of patients in the current study were motorcyclists followed by pedestrians and backseat passengers, commonly young males. This agreed with results of Alghnam et al., (2019) and Bittar et al., (2020). In addition, Arafa et al.,(2020) on their study on 518 drivers in Egypt, reported increased incidence of lapses and mistakes among drivers $<30$ years. This high prevalence of accidents among young drivers may be attributed to risky driving behavior, over speeding and driving in high-risk environment without wearing protective helmet and clothes (Yousaf et al., 2013). Male predominance in the current study specially among motorcyclists (who were exclusively male) was also observed by Sisimwo, et al.,(2014). Males tend to use motorcycles either to travel long distances to work, leisure and delivery commercial work (Yousaf et al., 2013), specially with increased delivery work during COVID19 lockdown (Zafri et al., 2021).

Samadhi and Ruwanpura, (2019) in accordance with our study, reported that day accidents were higher in motorcyclist and backseat passengers, while in our study, night accidents were higher only in pedestrians. In contrast to this, Chichom-Mefire et al., (2015) reported higher incidence of motorcycle accidents during nighttime. This difference in timing of the accidents was explained by Zhang and Hassan, (2019) who stated that some factors may affect incidence of accidents time such as the day of the week, unclear foggy weather conditions and detached asphalt surface in the daytime model. While factors like excessive speed, rainy weather, angle, and U-turn crashes are substantial in the nighttime model.

Frontal crashes hitting a vehicle or pedestrians in agriculture road were the main crash mechanism and location in the current study. This agrees with the results of Yousaf et al., (2013). While Alghnam et al., (2019) reported fall from motorcycle as the main mechanism of injuries in motorcycle injuries in Saudi Arabia. Sisimwo et al., (2014) found that number accidents are higher on highways. The main accident cause in this study was over speed. This came in accordance with previous related studies (Yousafet al., 2013; Fernando et al., 2017).The current study was carried out in El Gharbia governorate which lies midway along the agricultural road between the two 
major capitals of Egypt, Cairo and Alexandria. Higher rates of RTAs over this road can be explained by congested traffic over it, bad road conditions with poor road maintenance together with high speed reckless driving of some drivers.

Severity of injuries was higher in motorcyclist group than other groups. Samadhi and Ruwanpura, (2019) reported also higher injury severity in motorcyclist than backseat passengers. However, Chiang et al., (2014) reported no statistically significant difference in the ISS between drivers and backseat passengers. Injury severity are strongly corelate with lack of protective measures such as seat belts, airbags etc. and also the lesser stability of the motorcycle (Samadhi and Ruwanpura, 2019). In addition, the motorcyclist often absorbs all kinetic and compressive energy resulting from the crash. Even with minor collisions, they tend to receive serious injuries (Kudebong et al., 2011).

Craniofacial injuries were the most reported injuries in about $83 \%$ of patients in the current study and the commonest in all groups represented mainly with different facial injuries and meningeal hemorrhage. Previous studies described head injuries as the most reported category of injuries in motorcycle accidents (Fitzharris et al., 2009; Sisimwo et al., 2014; Granieri et al., 2020). Unlikely, Alghnam et al., (2019) and Samadhi and Ruwanpura, (2019) reported head injuries as the second most common injuries in motorcycle accidents after extremities injuries. Higher incidence of head injuries in motorcycle accidents because most or even all motorcyclist and backseat passengers did not pay attention to wearing helmets and other safety wears while riding. This would expose them to severe head injuries and high mortality (Weiss et al., 2010). It was ascertained by many researches that helmet usage would be able to decrease head and facial injuries to $50 \%$ and also decrease the severity of these injuries (CDC, 2012).

Abdominopelvic injuries were the second most common in motorcyclists. Similar previous results were reported by Liang et al., (2015). Splenic injuries and intraperitoneal free fluid were the mostly reported abdominopelvic injuries while Liang et al., (2015) reported hepatic injuries more common than splenic in motorcycle accidents. In backseat passengers and pedestrians, chest injuries came after head injuries. The most commonly reported chest injuries were lung contusion and pneumothorax. Contrary to this, Fitzharris et al., (2009) reported no or even very minimal differences between riders and backseat passengers in anatomical site and severity of injury. Upper limb fractures were more reported than lower limb fractures while Yousaf et al., (2013) reported higher incidence of lower limb injuries than upper limb in motorcycle accidents. Injuries to vital organs like liver, spleen and lung advocate the importance of their protection in motorcycle riders through wearing protective clothing in addition to helmets to reduce the mortality rates in this population.

In the presenting study, mortality rate was as high as $21 \%$. Ankarath et al., (2002) in United
Kingdom and Tan Chor Lip et al., (2019) in Malaysia reported lower mortality rates (about $6 \%$ and $9 \%$ respectively). Studies by Ankarath et al., (2002), and Nwadiaro et al., (2011) established that traumatic brain and abdominal injuries were the major reason for lower survival rates in these types of accidents.

In addition, $14 \%$ of patients in the presenting study have been discharged with permanent infirmity in the form of splenectomy, skull bone defect, nerve palsy or amputated limb and even combination of any of them in the same patient. DiMaio and DiMaio, (2001) reported that some motor cyclists to beheaded or having avulsed arms that completely amputated in motorcycle accidents. Alghnam et al., (2019) reported that about 9\% of patients injured in motorcycle crashes suffered amputation to a limb, mostly lower limb. Limbs usually stuck in the metal chain of the motorcycle. Limb amputation or any infirmity can affect physical ability in these young patients which may last a lifetime or need long periods of rehabilitation. Adding to the physical disability and economic burden, the significant psychological consequences of these injuries like anxiety and depression on the patients.

About $83 \%$ of patients needed ICU admission; the majority of them were motorcyclist. This result agrees with results of Alghnam et al., (2019) but in contrary to Chichom-Mefire et al., (2015) who reported ICU admission of only $6 \%$ and $9 \%$ of patients respectively). High incidence of head injuries together with visceral and chest injuries were the indicators of ICU admission.

Mean duration of hospital stay was 3.7 days and was significantly higher in motorcyclist than other groups. Duration of hospitalization is used as a tool of measuring the morbidity and outcome in trauma patients. Long hospitalization period is directly proportionate to trauma severity (Bittar et al., 2020). Prolonged hospitalization is associated in majority of cases by increased consumption of hospital properties together with decrease in productivity of population either due to time spent during hospitalization or due disability of traumatized victims. Hospitalization, in this study, was indicated to existence of head injuries, numerous fractures and other established co-morbidities of the patients. Majority of patients' management were conservative with either skeletal or skin traction, wound care, symptomatic supportive and observational follow up. While others were managed with operative interventions. In the evaluation of medical records, Bittar et al., (2020) in Brazil demonstrated in their study that the average time of hospital stay for motorcycle accidents patients was 9 days (1-20 days), with hospital costs about16, 307 dollar per patient.

Severity of injuries in relation to different factors was studied where, frontal impact, over speed, hitting a vehicle and agriculture road accidents were highly associated with major injuries. Yousaf et al., (2013) and Fernando et al., (2017) reported that over speed, reckless driving, lack of legal license together lack of helmet use and alcohol and drug illicit use as risk factors lead to severe injuries in motorcycle crashes. 


\section{Conclusion}

Motorcycle accidents are common in young and middle aged males. Day frontal crashes hitting a vehicle or pedestrians in agriculture road and over speed were the main circumstances of motorcycle accidents. Head injuries, abdominopelvic and chest injuries were the commonly reported injuries in the current study. Severity of injuries was higher in motorcyclists than other groups. Over speed, frontal impact at agricultural road together with absence of protective helmets and clothes were associated with major severe injuries and higher mortality in patients of motorcycle accidents.

\section{Recommendations}

The current study strongly recommends general public education program about road safety, application of traffic rules specially licensing and helmet use and upgrade of agricultural roads together with regular road maintenance.

\section{References}

Alghnam S, Alsulaim HA, BinMuneif YA, et al. (2019): Injuries following motorcycle crashes at a level-1 trauma center in Riyadh.Annals of Saudi Medicine, 39(3):185-191.

Ankarath S, Giannoudis PV, Barlow I,et al. (2002): Injury patterns associated with mortality following motorcycle crashes.Injury, 33(6):473-477.

Arafa A, Saleh LH and Senosy SA. (2020): Age-related differences in driving behaviors among nonprofessional drivers in Egypt.PLOS ONE, 15(9):e0238516.

Aristizábal D, González G, Suárez JF andRoldán P. (2012): Factors associated with fatal trauma in Medellín (Colombia) motorcyclists, Biomedica: Revista Del Instituto Nacional De Salud, 32(1):112-124.

Bittar CK, Cliquet A, Costa VSDAD, et al. (2020): Socioeconomic impact of motorcycle accident victims in the emergency room of a hospital (part 2). ActaOrtopédicaBrasileira, 28(3):149-151.

Carrasco CE, Godinho M, Berti de Azevedo Barros M,et al. (2012): Fatal motorcycle crashes: a serious public health problem in Brazil. World Journal of Emergency Surgery,7(1):S5.

CDC (Centers for Disease Control and Prevention) (2012): Helmet use among motorcyclists who died in crashes and economic cost savings associated with state motorcycle helmet laws-United States, 2008-2010. Morbidity and mortality weekly report, 61(23):425-430.

Chiang VX, Cheng JY, Zhang ZC and Teo LT (2014): Comparison of severity and pattern of injuries between motorcycle riders and their pillions: A matched study. Injury, 45(1):333-337.

Chichom-Mefire A, Atashili J, Tsiagadigui JG,et al. (2015): A prospective pilot cohort analysis of crash characteristics and pattern of injuries in riders and pillion backseat passengers involved in motorcycle crashes in an urban area in Cameroon: lessons for prevention. BMC Public Health, 15(1):915.
DiMaio D and DiMaio V (2001): Forensic Pathology. $2^{\text {nd }}$ ed. United Kingdom: CRC Press.

El Bakash OH, Kabbash AM, El Gohary MSA and Hafez ASAF (2016): Evaluation of the patterns of injuries in road traffic accidents in great cairo, egypt.The Egyptian Journal of Forensic Sciences and Applied Toxicology, 16(2):79-95.

Fernando DM, Tennakoon SU, Samaranayake AN and Wickramasinghe $\mathrm{M}(2017)$ : Characteristics of road traffic accident casualties admitted to a tertiary care hospital in Sri Lanka. Forensic Science, Medicine, and Pathology, 13(1): 4451.

Fitzharris M, Dandona R, Kumar GA andDandona L (2009): Crash characteristics and patterns of injury among hospitalized motorised twowheeled vehicle users in urban India.BMC public health, 9:11.

FoudaE, Youssef M,Emile S, et al. (2017): Pattern of major injuries after motorcycle accidents in Egypt: The Mansoura Emergency Hospital experience. Trauma, 19(1):39-45.

Granieri SS, Reitano EE, Bindi FF, et al. (2020): Motorcycle-related trauma:effects of age and site of injuries on mortality. A single-center, retrospective study. World journal of emergency surgery, 15(1): 18 .

Hinkle D, Wiersma W and Jurs SG (2003):Applied Statistics for the Behavioral Sciences. $5^{\text {th }}$ ed. Boston: Houghton Mifflin.

Jackson TL and Mello MJ (2013): Injury patterns and severity among motorcyclists treated in US emergency departments, 2001-2008: a comparison of younger and older riders. Injury Prevention: Journal of the International Society for Child and Adolescent Injury Prevention, 19(5): 297-302.

Javali RH, Krishnamoorthy, Patil A,et al. (2019): Comparison of Injury Severity Score, New Injury Severity Score, Revised Trauma Score and Trauma and Injury Severity Score for Mortality Prediction in Elderly Trauma Patients. Indian Journal of Critical Care Medicine: PeerReviewed, Official Publication of Indian Society of Critical Care Medicine, 23(2):73-77.

Kudebong M, Wurapa F, Nonvignon J, et al. (2011): Economic burden of motorcycle accidents in Northern Ghana. Ghana Medical Journal, 45(4):135-142.

Liang CC, Liu HT, Rau CS, et al. (2015): Motorcyclerelated hospitalization of adolescents in a Level I trauma center in southern Taiwan: a crosssectional study.BMC Pediatrics, 15(1):105.

Nwadiaro HC, Ekwe KK, Akpayak IC, Shitta H (2011): Motorcycle injuries in north-central Nigeria.Nigerian Journal of Clinical Practice, 14(2): 186 .

Oluwadiya KS, Kolawole IK, Adegbehingbe OO,et al. (2009): Motorcycle crash characteristics in Nigeria: implication for control.Accident; Analysis and Prevention, 41(2):294-298. 
Samadhi DAH and Ruwanpura PR (2019): Analysis of Injury Patterns of Motorcycle Riders Admitted to Teaching Hospital, Karapitiya, Sri Lanka.Medico-Legal Journal of Sri Lanka, 7(2):42.

Sanyang E, Peek-Asa C, Bass P, et al. (2017): Risk Factors for Road Traffic Injuries among Different Road Users in the Gambia. Journal of Environmental and Public Health: 8612953.

Sisimwo PK, Mwaniki PK and Bii C (2014): Crash characteristics and injury patterns among commercial motorcycle users attending Kitale level IV district hospital, Kenya. Pan African Medical Journal, 19.

Tan Chor Lip H, Tan JH, Mohamad Y, et al. (2019): Clinical characteristics of 1653 injured motorcyclists and factors that predict mortality from motorcycle crashes in Malaysia.Chinese Journal of Traumatology, 22(2):69-74.
Weiss H, Agimi Y and Steiner C (2010): Youth motorcycle-related brain injury by state helmet law type: United States, 2005-2007.Pediatrics, 126(6):1149-1155.

WHO (2018): Global status report on road safety 2018. Available at: https://www.who.int/newsroom/fact-sheets/detail/road-traffic-injuries. accessed at 20 March 2021.

Yousaf M, Iqbal M, Akram Mand Choudhary R (2013): Pattern of orthopaedic injuries in motorcycle accidents.Annals of Punjab Medical College, 7(1):77-84.

Zafri N, Khan A, Jamal Sh and AlamB (2021): Impact of COVID-19 Pandemic on Motorcycle Purchase in Dhaka, Bangladesh.Frontiers in Future Transportation, 2: 646664.

Zhang K and Hassan M (2019): Crash severity analysis of nighttime and daytime highway work zone crashes.PLOS ONE., 14(8): e0221128.

\section{أنماط وشده الإصابات في مصابي حوادث الدراجات الناريه بمستثفي الطوارئ الجامعي بطنطا}

ناديه عزت هلال ا و محمد عبد الهادى شامه' وسماح ماهر البسطويسى'

\section{الملخص العربى}

مقدمة: في السنوات القليلة الماضية ،تبين إزدياد استخدام الدراجات النارية على نطاق واسع إما كوسيلة للتنقل أو للتجارة وتوصيل الطلبات للمحال والمنازل

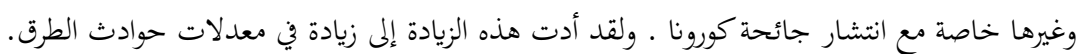

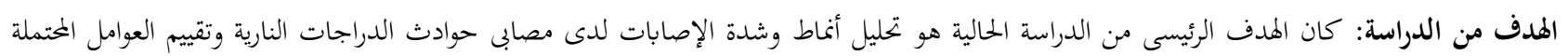
المرتبطة بشدة تلك الإصابات.

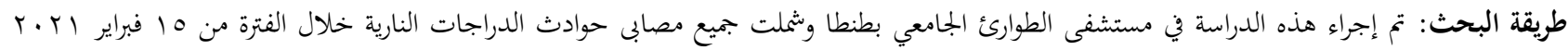

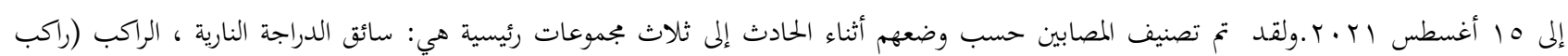
المقعد الخلفي للدراجات النارية) أو المشاة. وتم تسجيل البيانات الشخصية الخاصة بالمصابين وكذلك ظروف وأليه وأسباب التصادم ونمط وشدة الإصابات بالإضافة إلى نتائج الإصابة لمؤلاء المصابين.

النتائج: أغلب حوادث الدراجات النارية في هذه الد راسة حدثت لمن هم في منتصف العمر من الذكور. بالإضافة إلى أن التصادم الأمامي بالسيارات أو بالمشاة

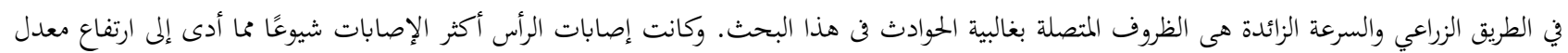

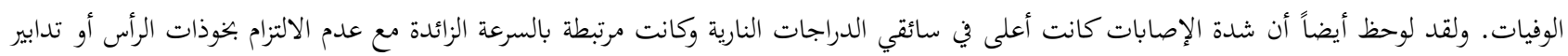
الحماية الشخصية. الإستنتاجات: تظهر الدراسة الحالية أن إصابات الرأس كانت أكثر أنواع الإصابات شيوعًا بين مرضى حوادث الدراجات النارية.وأن هناك إرتباطا وثيقا بين شدة

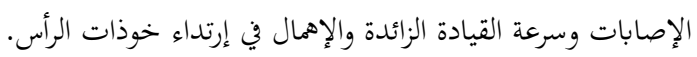

